\title{
Die Milchdrüse thyreopriver Ziegen ${ }^{1}$.
}

\author{
Von \\ Privatdozent Dr. Alfred Trautmann.
}

(Aus dem physiologischen Institute der Tierärztlichen Hochschule zu Dresden.

Direktor: Geh. Rat Prof. Dr. med. et med. vet. et phil. Ellenberger.)

Mit 3 Textabbildungen und 3 Abbildungen auf Tafel II und III.

(Eingegangen am 3. Julli 1919.)

Gelegentlich von Untersuchungen, die sich mit den nach der Thyreoidektomie auftretenden krankhaften Erscheinungen beschäftigten, wurde im physiologischen Institut der Tierärztlichen Hochschule zu Dresden von Zietzschmann (Beiträge zum Studium der Folgen der Thyreoidektomie bei Ziegen. Arch. f. wiss. Tierhlkd. 33. 1907 und Ein Beitrag zum Studium der Folgen der Schilddrüsenexstirpation, Mitt. Grenzgebieten der Medizin 19. 1908) und später von mir (Hypophyse und Thyreoidektomie. Frankfurt. Zeitschr. f. Pathologie 18. 1916) bei Ziegen festgestellt, dass auch die Milchdrüsen in ganz besonderer Weise klinisch zu beobachtende Erscheinungen aufwiesen, die nur als Folgen der Schilddrüsenexstirpation zu deuten waren. Bei thyreoidektomierten Ziegen, die in der Laktation standen, trat schon sehr bald nach der Operation ein Nachlassen ${ }^{2}$ ) in der Menge der abgesonderten. Milch ein, das weiter anhielt und das sich bei den einzelnen Individuen in verschiedener Art äusserte. Hand in Hand mit der Milchabnahme ging eine Veränderung der Farbe und der Zusammensetzung der Milch; die Milch nahm mit der Zeit einen stark gelblichen Ton an, um kurz vor dem Eintritt der Agalaktie einem schmutzig grauen Platz zu machen; ausserdem trat mit der Milchabnahme eine Atrophie der Mamma und der Zitzen ein, die sich nach der Agalaktie noch in grossem Maasse steigerte. Die Oberfläche des Euters und der Zitzen war dann mit seborrhoeischen, dicken Auflagerungen vollkommen bedeckt und zeigte stets ein runzeliges, welkes Aussehen.

1) Die nachstehenden Ausführungen sind das Ergebnis von Untersuchungen, die in unserem Institut über den in Frage stehenden Gegenstand angestellt wurden. Infolge des Krieges konnte erst in letzter Zeit das von mir gesammelte reiche, bisher nur zum Teil bearbeitete Material weiter ausgiebig verwertet und dadurch die von mir in einer früheren Arbeit (Frankf. Zeitschr. f. Path. 1916) niedergelegten Befunde wesentlich erweitert worden.

2) Nach Zietzschmanns Angabe hat auch Lanz (Progenitur thyreopriver. Arch. f. klin. Chir. Bd. 74. 1904) äbnliche Ferhältnisse festgestellt. 
Bei Ziegen, dje lange Zeit nach der Operation getötet wurden, konnte festgestellt werden, dass stellenweise die Zitzen um drei Viertel ihrer Länge verkürzt waren. Diese in unserem Institute an der Milchdrüse gemachten klinischen Befunde wiesen darauf hin, die Struktur der Mamma nach der Thyreoidektomie zu untersuchen.

Die Untersuchungen wurden an den Milchdrüsen thyreoidektomierter Ziegen vorgenommen; das Material entstammt den gleichen Tieren, die ich vor einigen Jahren bei meinen Untersuchungen über die klinischen Folgeerscheinungen der Thyreoidektomie und den Untersuchungen über die strukturelle Veränderung der Hypophyse nach der Schilddrüsenexstirpation verwandt habe.

Infolge der sinnfälligen Veränderungen, die bei meinen Untersuchungen bei Ziegen nach der Thyreoidektomie sowohl bezüglich der Milchmenge wie der Milchbeschaffenheit erkannt wurden, bestand von Anfang an die Absicht, die Milch thyreoidektomierter Ziegen zu analysieren und die veränderten Sekretionsverhältnisse näher zu studieren. Die von der chemisch-physiologischen Abteilung unseres Instituts nach dieser Richtung in Aussicht genommenen Arbeiten mussten infolge des Krieges unterbleiben. Inzwischen ist von dem früheren Assistenten der chemisch-physiologischen Versuchsstation des physiologischen Instituts der Tierärztlichen Hochschule zu Dresden, jetzt an dem Landwirtschaftlichen Institut der Universität Königsberg befindlichen Herrn Professor Dr. Grim mer, der die Veränderungen in der Milchsekretion thyreopriver Ziegen bei meinen Untersuchungen zu beobachten reichlich Gelegenheit hatte, in begrüssenswerter Weise die Bearbeitung dieser Frage in Angriff genommen worden.

Die vorliegenden, in der biochemischen Zeitschrift Bd. 48, 1918 veröffentlichten Untersuchungen Grimmer's, „Beiträge zur Milch schilddrüsenloser Ziegen", sind als der Anfang einer grösseren Reihe von Versuchen, deren Fortführung beabsichtigt ist, zu betrachten. Vorläufig wurden von. Grimmer nur an eines Ziege Untersuchungen angestellt. Die Untersuchung bei diesem Tiere bezog sich auf die Wägung der Milchmenge in bestimmten Zeitabschnitten, die Bestimmung des spezifischen Gewichtes, des Fettgehaltes und der Gesamtanalyse der Milch. Die Laktationsperiode wurde durch die Thyreoidektomie in zwei annähernd gleich grosse Zeitabschnitte zerlegt, deren erster die Zusammensetzung der Milch unter normalen Verhältnissen, deren zweiter die Folgen der Operation auf die Milchsekretionsverhältnisse widerspiegelt.

Gleich mir hat auch Grimmer als Folgen der Thyreoidektomie zunächst eine rapide Abnahme der Milchmenge beobachtet, die er wohl mit Recht als eine Reaktion auf die Operation an sich zu betrachten geneigt ist, und die mit dem eigentlichen Ausfall der Funktion der Schilddrüse 
nichts zu tun haben dürfte; dies folgt schon daraus, dass nach węnigen Tagen sich die Milchmenge wieder steigerte. Erst nach der zweiten Woche nach der Operation sank sie ziemlich rasch und gleichmässig.

Hinsichtlich der Zusammensetzung der Milch änderte sich zunächst noch sehr wenig. 6 Wochen nach der Schilddrüsenexstirpation wurde die Milch hinsichtlich ihres Fett- und Stickstoffgehaltes wesentlich gehaltreicher als vorher, während der Gehalt an Milchzucker im grossen und ganzen unverändert blieb. Die einzige sofort eintretende Veränderung erlitt der Aschengehalt der Milch, der bis zum Laktationsende erhöht blieb. Der prozentische Phosphorsäuregehalt der Asche blieb bis zum Ende der Laktation dauernd wesentlich erhöht, während der Kalkgehalt der Asche sich nur geringfügig über die Norm erhob. Ausserordentlich geringfügig waren die Veränderungen, die in der Verteilung des Stickstoffs auf die einzelnen Bestandteile zum Ausdruck kamen. Die Reaktion der Milch wurde durch die Thyreoidektomie nicht unwesentlich beeinflusst. Die normalerweise nur geringe Azidität stieg und erhielt sich nach achtwöchentlichem Bestehen des thyreopriven Zustandes fast dauernd auf 9 Säuregraden (nach Soxhlet-Henkel). Das plötzliche Ausbleiben der Peroxydasenreaktion mit Guajaktinktur etwa von der sechsten Woche nach der Operation an und der im Laufe der Zeit in viel schwächerem Maasse auftretenden Rothenfuse $r$ 'schen und Benzidinreaktion der Milch wie auch die Änderung in der Zusammensetzung der Milchasche deuten im vorliegenden Falle auf eine zweifelsfreie, wenn auch geringgradige Funktionsstörung der Milchdrüse infolge Schilddrüsenexstirpation hin.

Da die Milch jedoch bis zum Schlusse der Laktation ihr normales Aussehen, das sie vor der Operation aufwies, behielt und nicht die an den meisten der von mir verwandten Ziegen, von Grimmer selbst gesehene, verschiedene äussere Beschaffenheit der normalen Milch annahm, so glaubt Grimmer, dass mit grosser Wahrscheinlichkeit anzunehmen ist, dass bei der operierten, aber aus bestimmten Gründen noch nicht getöteten Ziege akzessorische Schilddrüsen vorhanden sein werden, die durch Hypertrophie kompensatorisch für die operativ entfernten eingetreten sind. Das würde ganz meinen Befunden entsprechen; denn ich stellte fest, dass bei Ziegen, die längere Zeit nach der Thyreoidektomie reichlicher Milch von normalem Aussehen sezernierten, bei der Sektion regelmässig Glandulae thyreoideae accessoriae vorhanden waren. Dass bei Ziegen akzessorische Schilddrüsen häufig vorkommen, ist eine Tatsache, die ausser von mir auch früher schon von Zietzschmann festgestellt wurde. Interessant wäre es, zu wissen, ob sich in der Zeit, in der die Milch nach der Thyreoidektomie untersucht wurde, an dem Grimmer'schen Versuchstiere anch 
klinische Folgeerscheinungen gezeigt haben, wodurch die Grimmer'sche Vermutung sich zum Teil bekräftigen liesse.

Weitere Untersuchungen der Zusammensetzung der Milch thyreoidektomierter Tiere sind zurzeit nicht vorhanden, desgleichen konnten auch keine sonsti-

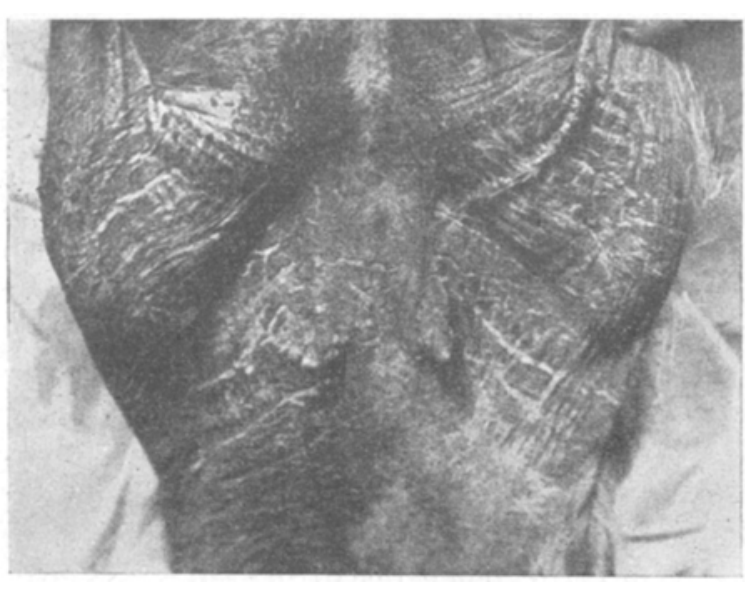

Abb. 1. Mammargegend einer Ziege 27 Monate nach der Thyreoidektomie. gen Angaben in der Literatur über die strukturelle Veränderung der Milchdrüse nach derThyroeidektomie gefunden werden.

An den Milchdrüsen thyreoidektomierter Ziegen konnte folgendes festgestelltwerden: Nach der Schilddrüsenexstirpation tritt bei Ziegen, die in der Laktation stehen, sehr bald ein erhebiicher Abfall der Milchproduktion ein. Die Agalaktie entsteht viel früher als bei nicht thyreopriven Tieren. Hand in Hand mit dem
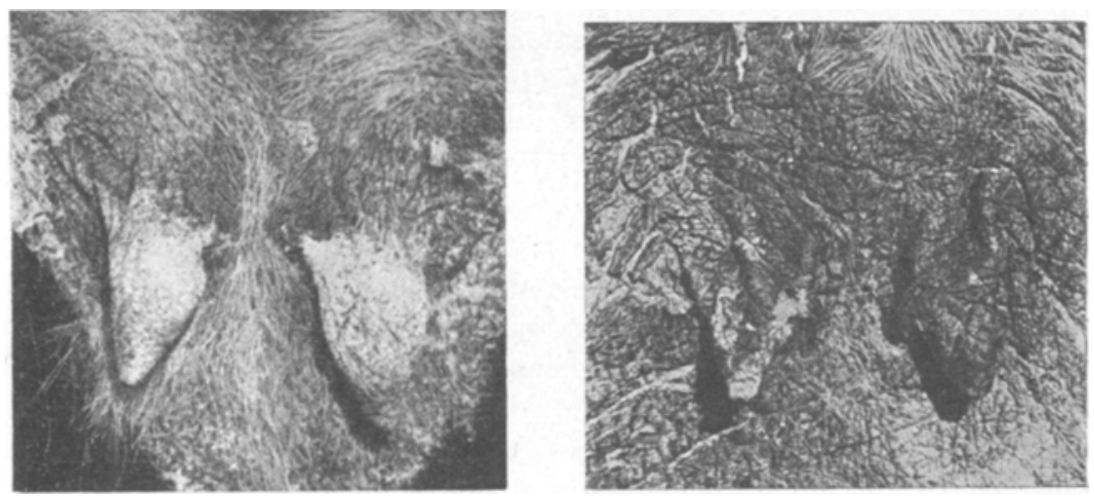

Abb. 2. Mamma von Ziegen 10 Monate (A) und 27 Monate (B) nach der Thyreoidektomie. Abbildung $B$ ist eine stärkere Vergrösserung der Abb. 1.

raschen und erheblichen Abfall der Menge der täglich produzierten Milch geht eine Veränderung in der Farbe und Zusammensetzung der Milch. Neben der Minderung der Milehisekretion lässt sich eine 
Atrophie der Mamma und ihrer Zitzen erkennen, die sich nach Eintritt der Agalaktie noch erheblich steigert. Der atrophische Zustand des Euters kann so weit fortschreiten, dass die sonst sackartig zwischen beiden Hinterschenkeln tief herabhängende, deutlich sichtbare Milchdrüse samt Zitzen so weit reduziert wird, dass sie der Bauchwand dergestalt anliegt, dass weder von der Seite noch von hinten ein Euter sichtbar ist. Die Oberfläche der Mammagegend zeigt in solchen Fällen ein welkes, runzeliges Aussehen, die Zitzen sind erheblich verkürzt, die Oberfläche des Euters und der Zitzen ist dann mit zum Teil starken seborrhosischen Auflagerungen bedeckt (Abb. 1 und. $2 \mathrm{~A}$ und $\mathrm{B})$.

Während bei Ziegen, bei denen der thyreoprive Zustand kürzere Zeit besteht, das makroskopische Aussehen und die Struktur der freigelegten Milchdrüse und ihres Parenchyms, soweit sie mit blossen Augen erkennbar ist, an der Oberfläche und auf Schnittflächen sich nicht wesentlich von dem normalen Verhalten unterscheidet, bietet die Betrachtung der Schnittfläche durch das Euter von Ziegen, welche längere Zeit ( 8 Monate und mehr) nach der Thyreoidektomie getötet und untersucht wurden, ein erheblich anderes als normales Aussehen. In solchen Fällen ist die Läppchenbildung des Organs nur undeutlich und verwischt oder gar nicht zu erkennen. Die normale gelblich-weisse Farbe des Parenchyms hat sich in der Regel in einen mehr grau-weissen Ton verwandelt. Die normaliter deutlich körnige Beschaffenheit des sezernierenden Milchdrüsengewebes ist gänzlich verschwunden. Die straffe, festweiche Konsistenz des normalen, durch das Sekret feuchten Gewebes hat einem verschieden weichen, fast trockenen Platz gemacht. Man glaubt, eine bindegewebige Masse vor sich zu haben, die von Kanälen, den Ausführungsgängen, durchzogen ist. Die Zisternen- und Zitzenschleimhaut liegt stark in Falten und ist mit schuppenartigen Häutchen bedeckt.

Mikroskopisch zeigt das Milchdrüsengewebe von laktierenden Tieren, bei denen der thyreoprive Zustand erst kürzere Zeit bestanden hat, aber eize deutliche Verminderung der täglich sezernierten Milchmenge während des Lebens der Tiere festzustellen war, neben normalen Läppchen von dem bekannten Aufbau (TafelIT Abb. 1 a) solche, die durch eine ausserordentlich grosse, in den Alveolen gelegene Anzahl von Kon. krementen auffallen (Abb. 2b-f). Manche Läppchen sind geradezu übersät mit diesen Bildungen. In der normalen sezernierenden Milchdrüse lassen sich zwar auch manchmal einzelne solcher Gerinnsel, die als Kaseiokonkremente aufzufassen sind, feststellen, und zwar dann, wenn die Entleerung der Drüse durch irgendwelche Umstände (psychische Erregungen usw.) auch nur während eines Tages ver- 
langsamt oder plötzlich unterbrochen wird. Nach der Thyreoidektomie trete. 1 diese Gebilde aber selbst bei gewissenbaftester Behandlung des Euters und sorgfältigster Ausführung des Melkaktes immer in erheblicher Zahl auf. In einzelnen Milchdrüsenläppehen sind sie nur vereinzelt, in anderen dagegen massenhaft zu finden. Die Konkremente, die durch irgendwelche unbekannte, durch die Schilddrüsenexstirpation verursachten Vorgänge, wahrscheinlich infolge Eindickung des Milchsekretes oder spärlicher Sekretion von wässerigen Bestandteilen in die sie anfangs nicht ganz erfüllenden Alveolen entstehen, werden entweder von dem. Alveolarepithel oder direkt vom Bindegewebe umgeben. In ersterem Falle sind die mehr oder weniger von den Konkrementen gedrückten Drüsenzellen im mikroskopischen Bilde teils in der ganzen Zirkumferenz der Konkremente erhalten. (Fig. 2c, $c^{\prime}$ ) und können die verschiedensten Gestalten von der niedrig-zylindrischen bis ganz platten Form aufweisen, teils trifft man solche nur auf einer kürzzeren oder längeren Strecke $\left(A b b .2 d, d^{\prime}\right)$ oder nur vereinzelt an. Wenn sie ganz fehlen, dann werden die Konkremente von dem perialveolären Bindegewebe (der Alveolenhülle) und dem Interalveolargewebe in der Regel lamellenartig umhüllt. Es ist dann auch die Membrana propria der Alveolen ganz oder zum Teil geschwunden. Die Grösse der Konkremente ist ganz verschieden. Man begegnet hier und da sehr grossen Bildungen und daneben kleineren und ganz kleinen. In bezug auf ihre Gestalt ist zu bemerken, dass in der Regel kugelige und eiförmige Formen vorherrschen, daneben finden sich aber auch ganz unregelmässige Gestalten, namentlich dann, wenn das Alveolarepithel nicht mehr erhalten ist, und wenn nach der Schilddrüsenexstirpation bereits eine längere Zeit vergangen ist. Manche dieser Bildungen ähneln dann bezüglich der Beschaffenheit der Oberfläche einer unregelmässig gestalteten Brombeere. Die Konkremente zeigen eine starke Neigung, sich mit basischen Farbstoffen, zum Beispiel mit Haematoxylin, zu tingieren. In der Affinität zu den Farbstoffen bestehen viele Übergänge. Eine besonders intensive Cyanophilie weisen die direkt vom Bindegewebe ohne Vorhandensein von Alveolarepithel umhüllten, längere Zeit bestehenden Konkremente auf. Ein Teil der Konkremente erscheint vollständig homogen und zeigt überhaupt keine Struktur, andere lassen eine hellere zentrale Partie und eine dunkler gefärbte periphere Schicht oder umgekehrt erkennen, wieder andere sind konzentrisch geschichtet; auch finden sich solche, in denen heller und dunkler tingierte Zonen regellos abwechseln.

Die Läppchen, in denen diese Konkremente in grösserer Zah] auftreten (Tafel II Abb. 1b), machen nicht den Eindruck des Gewebes, das in der normalen laktierenden Milchdrüse angetroffen wird. Bei der Anwesenheit grösserer Konkremente sind die Alveolen 
unverhältnismässig stark erweitert oder zystisch aufgetrieben. Der durch die dauernde Vergrösserung der Konkremente auf das Alveolenepithel ausgeübte Druck bedingt eine Abplattung und Atrophie der Zellen and schliesslich ein volles Schwinden der gesamten Alveolenwand. In solchen Läppchen treffen wir bei längerer Dauer des thyreoidektomierten Zustandes viele Stellen, an denen die Struktur und Textur der Drüse vollkommen verschwunden ist. Es finden sich dann an verschiedenen Stellen der Läppchen einzelne zugrunde gehende, einst die konkrementhaltigen Alveolen auskleidende Zellen oder Gruppen solcher, an deren Stelle später mit Leukocyten durchsetztes Bindegewebe tritt. Durch die auf diese und andere Weise entstandene Vermëhrung des intralobulären Bindegewebes wird von diesem, durch reichliches, zelliges Infiltrat ausgezeichnetes Interalveolargewebe andauernd ein Druck auf die noch vorhandenen normalen Alveolen. der Läppchen ausgeübt; die Alveolen werden dadurch verkleinert, obliterieren, stellen ihre milchbildende Funktion ein und gehen schliesslich zugrunde. Man findet deshalb in einem, an Konkrementen reichen Läppchen neben einzelnen, durch Konkremente erweiterten Alveolen auch Alveolen, die sehr klein sind und recht oft überhaupt kein Lumen erkennen lassen. An den niedrigen und unregelmässig gestalteten Zellen dieser Alveolen sind Zeichen irgendwelcher Funktionstätigkeit nicht wahınehmbar. Im Gegenteil lassen an solchen Stellen die oft pyknotischen Kerne der Milchdrüsenzellen auf allmählichen, histolytischen Zerfall der Zellen schliessen. Der für die tätigen Milchdrüsenzellen so spezifische und charakteristische Vorgang der Fettbildung und Ablagerung der Fettkörnchen in den Drüsenzellen geht in den von der beschriebenen Alteration ergriffenen Läppchen gänzlich verloren.

Durch die Vermehrung des interalveolären (intralobulären) und darauf auch des interlobulären Bindegewebes wird das Parenchym der Milchdrüsenläppchen atrophisch. Das Interstitialgewebe zeigt somit eine Zunahme auf Kosten des Parenchyms. Mit der längeren Dauer des thyreopriven Zustandes werden immer mehr Lobuli ergriffen. Man begegnet dann neben wenigen Milchdrüsenläppchen von annähernd normalem Bau einer grossen Anzahl solcher Läppchen, die sich durch geringe Ausdehnung, also durch ihre Kleinheit, auszeichnen, durch viel interstitielles, an elastischen Fasern und Fettgewebe armes Bindegewebe getrennt sind und somit weit auseinander liegen. Diese Läppchen lassen die ursprüngliche drüsige Struktur vermissen; diese wird auch durch die zahlreich intraepithelial, interalveolär und interlobulär auftretenden Leukocyten verwischt, deren überreichliches Vorkommen in der laktierenden Milchdrüse bei nicht normalen Zuständen der Drüse oft vorkommt. Wenn noch reichlicher Milch abgesondert 
wird, dann sind immer einige Läppchen von normalem Bau im Milchdrüsengewebe vorhanden. Beim Herabgehen der Milchproduktion auf wenige Kubikzentimeter pro Tag - ein Zustand, der durchschnittlich immer relativ schnell eintritt - sind dagegen ganz normale Drüsenläppchen kaum noch anzutreffen. Fast immer sind scheinbar normal sich präsentierende Lobuli dennoch in irgendeinem Abschnitte alteriert. In einem Teil der Lichtungen der Alveolen scheinbar normaler Läppchen, wie auch intraepithelial trifft man nicht selten eine reiche Zahl immigrierter Leukocyten an.

Die intralobulär gelegenen Zentralräume (Sekretsammelräume) bzw. -gänge und die interlobulär liegenden Ausführungsgänge weichen im Gewebe des laktierenden Euters thyreoidektomierter Ziegen am wenigsten von der normalen Beschaffenheit ab. Die mehrfach erwähnten und beschriebenen Konkremente finden sich niemals in deren Lumen, dagegen aber finden sich in ihren Hohlräumen, namentlich nach längerem Bestehen der Schilddrüsenexstirpation, Haufen abgelöster Drüsenepithelien und leukocytärer Elemente (Tafel II Abb. 2a). Letztere lagern in diffuser Verteilung auch oft in dem subepithelialen Gewebe der Zentral- und Ausführungsgänge (Tafel II Abb, 3a, c).

Nach Fintritt des agalaktischen Zustandes der Milchdrüse, welcher sich bei thyreoidektomierten Ziegen in der Regel bei weitem früher einstellt als bei normalen Ziegen, steigert sich der atrophische Zustand des Parenchyms in hohem Maasse. Man begegnet dann in den mikroskopischen Bildern niemàls mehr Milchdrüsenläppchen, deren Alveolarevithel irgendwelche sekretorische Erscheinungen anfweist (Abb. 2, 3). Die Läppchen, welche auch ein ganz anderes Aussehen als die einer normalen, trocken stehenden Ziege haben, werden noch kleiner, das interalveoläre, leukocytenreiche Bindegewebe vermehrt sich immer mehr; deutlich alveoläre Bildungen sind im Läppchen nur noch ab und zu, also sehr selten, zu bemerken, bis schliesslich bei langem Leben thyreoidektomierter agalaktischer Ziegen ein Bild resultiert, in welchem die zum Teil noch erhaltenen, aber atrophierenden intralobulären Zentralgänge, umgeben von leukocytenreichem Bindegewebe und einzelnen kleinen Alveolen oder Alveolarzellresten die früher vorhandenen Läppchen noch andeuten (Abb. 3b).

Konkrementen (Abb. 2c), die sich durch einen relativ festen Aggregatzustand auszeichnen, begegnet man in den infolge der Schilddrüsenexstirpation agalaktischen Eutern nicht mehr in solcher Menge wie in noch laktierenden Milchdrüsen. Das ist ja auch verständlich, weil einmal infolge des Mangels an normalen sezernierenden Milchdrüsenläppchen Konkremente nicht mehr gebildet werden, und weil ferner die in der Laktationsperiode entstandenen teilweise resorbiert werden. Die in solchen Drüsen noch vorkommenden selteneu Konkremente 
sind in den meisten Fällen nicht mehr von den Epithelzellen der Drüsenalveolen umgeben, sondern liegen frei im interalveolären Bindegewebe. Ausserdem ist ihre Grösse, da sie durch Resorption verkleinert wurden, nicht mehr so erheblich wie bei Tieren, bei welchen der thyreoprive Zustand nicht so lange Zeit bestanden hat und die Milchdrüse noch sezernierte. Infolge der beginnenden Resorption der Konkrementsubstanz, die an den einzelnen Stellen der Konkremente verschiedengradig abläuft, trifft man in Drüsen solcher Tiere viel häufiger als in der laktierenden Milchdrüse thyreoidektomierter Ziegen fast keine kugeligen Konkrementformen mehr, sondern solche von den verschiedensten Formen mit ganz ungleichmässigem Tinktionsvermögen. Längere Zeit nach der Schilddrüsenexstirpation (20 Monate und mehr) trifft man überhaupt keine Konkremente mehr an.

Das interstitielle, interlobuläre Bindegewebe ist in nicht mehr laktierenden Milchdrüsen stark entwickelt (Abb. 2, 3d). Es ist fast frei von elastischen Gewebselementen und Fettzellen. Um die Gefässe liegen starke adventitielle, bindegewebige Scheiden (Abb. $2 d^{\prime}$ ). Die Milchdrüsen von thyreoidektomierten, längere Zeit nicht mehr laktierenden Ziegen machen einen stark cirrhotischen Eindruck.

An den Zitzen thyreoidektomierter Ziegen fallen, besonders lange Zeit nach der Operation, am Integumentblatte die Zeichen der Seborrhoe auf. Die Zisterne verhält sich strukturell ähnlich wie die Ausführungsgänge.

Von ganz besonderem Interesse ist die Tatsache, dass an einem schwangeren Tiere sich Zeichen der Vergrösserung des Euters weder während bzw. gegen Ende der Schwangerschaft noch auch nach der Geburt bemerkbar machten. Das Euter dieses Tieres, welches 20 Tage nach der Geburt eines 24 Stunden post partem gestorbenen kretinoiden Jungen nur $10-20 \mathrm{ccm}$ Milch pro Tag sezernierte, wies neben dem stark ausgeprägten bindegewebigen inter- und intralobulären Stützgerüst fast nur Läppchen mit in der Ruhe befindlichen Alveolen auf. Alveolen mit tätigen Zellen waren nur in geringer Menge vorhanden. Ihnen fehlten áber die bei normalen Verhältnissen reichlich in dən Drüsenzellen vorhandenen Fettkörnchen fast ganz, wie auch in dem im Lumen der Alveoli befindlichen Sekrete fast gar keine Fettröpfchen zu sehen waren. Besonders zahlreich waren Kaseinkonkremente vorhanden. Der thyreoprive Zustand der betreffenden Ziege und die schon während der Schwangersehaft eingetretenen Strukturveränderungen der Milchdrüse haben offenbar das Eintreten der Sekretionsvorgänge an den Zellen und der mit ihnen einhergehenden hypertrophischen Verhältnisse nicht zugelassen. In diesem Zusammenhang ist nicht uninteressant, darauf hinzuweises, dass von mir öfter beobachtet wurde, dass thyreoidektomierte Ziegen nur selten brünstig wurden 
und konzipiertar, und dass sie, wenn letzteres eintrat, in der Regel abortierten.

Auf die Milchdrüse jungfräulicher Tiere hat die Thyreoidektomie, sowert aus dem vorhandenen, in dieser Richtung geringen Material geschlossen werden kann, keinen Einfluss. Wenigstens konnten nach der gleichzeitigen Tötung von drei Ziegen eines Wurfes, von welchen bei der einen Zieg > die Schilddrüse total, bei del zweiten nur der Lobus dexter thyreoideae entfernt wurde, die dritte aber als Kontrolltier diente, keine Unterschiede in der Struktur der Milchdrüse festgestellt werden.

Weiter ist bemerkenswert, dass bei thyreoidektomierten Ziegen, bei denen sich bei der Sektion Glandulae thyreoideae accessoriae von wesentlichem Umfanga vorfanden, in der histologischen Struktur der Milchdrüse keineilei Abweichungen von dem normalen Verhalten sich feststellen liessen. Die Milchdrüse solcher Tiere sezernierte in gewöhnlicher Weise. Die Agalaktie stellte sich unter den gleichen Verhältnissen wie bei nicht operierten Tieren ein.

Aus den Untersuchungen scheint weiter hervorzugehen, dass bei älteren laktierenden Tieren, bei denen nach meinen Befunden im allgemeinen der Abfall der täglich produzierter Milchinenge schneller in die Erscheinung tritt als bei jüngeren in der Laktation stehenden, die oben erwähnten Alterationen in der Struktur sich früher erkennen lassen und auch schneller und intensiver fortschreiten als bei jungen Tieren. Bei erwachsenen gleichaltrigen Tieren ist der Umfang des veränderten Milchdrüsengewebes verschieden. Es besteht kein gesetzmässiges Verhalten in der Weise, dass die Grösse der Veränderungen von dex Länge der nach der Thyreoidektomie verstrichenen Zeit abhängt, dass also die am spätesten nach der Thyreoidektomie zur Untersuchung gelangte Milchdrüse die grössten Alterationen zeigt. Jedoch ist immer bei längerem Bestehen der Schilddrüsenexstirpation, zumal wenn die Tieie nicht mehr laktierten, die Reaktion der Milchdrüse auf die Thyreoidektomie erheblich. Auch bei gleichaltrigen, nach. gleicher Zeit nach der Thyreoidektomie getöteten Tieren ist das mikroskopische Bild nicht gleichartig. Bei einem Tier erfolgt die Reduktion des Parenchyms der Mamma schneller und ausgiebiger als bei einem anderen. Es bestehen somit erhebliche individuelle Verschiedenheiten in bezug auf die nach der Thyreoidektomie eintretenden Folgen, und zwar bei der Milchdrüse namentlich in bezug auf die Schnelligkeit des Eintritts und die Grösse der reaktiv nach der Thyreoidektomie eintretenden strukturellen Veränderungen. Es besteht aber kein Zweifel, dass mit der Länge der Zeit bei jeder thyreoidektomierten Ziege die In- und Extensität der Strukturveränderungen gleichmässig. 
zunehmen. Zwischen dem Ende des Auftretens der nach der Thyreoidektomie wahrnehmbaren klinischen Erscheinungen und der Intensität der in der Mamma sich vorfindenden Veränderungen lassen sich keine Parallelen ziehen. Immerhin lässt sich sagen, dass bei einer thyreopriven Ziege mit hochgradigen klinischen Erscheinungen auch die Struktur dər Milchdrüse ganz wesentliche Zeichen von Atrophie des Drüsengewebes aufweist, zumal wenn die Athyrie längere Zeit bestanden hat. Ziegen, denen nach der Thyreoidektomie Thyreoidintabletten verabreicht wurden, lassen vermutlich nicht so schnell und nicht so erhebliche Veränderungen des Milchdrüsengewebes erkennen wie solche, denen keine Tabletten gegeben werden.

Aus den oben angeführten Alterationen, die nach der Thyreoidektomie in der Milchdrüse entstehen, lässt sich schliessen, dass der Ausfall der Funktion der Thyreoidea schädigend auf dio Struktur der laktierenden Milchdrüse und damit auf ibre Funktion wirkt. Die in ihr ablaufenden Veränderungen sind degenerativer Natur. 
a
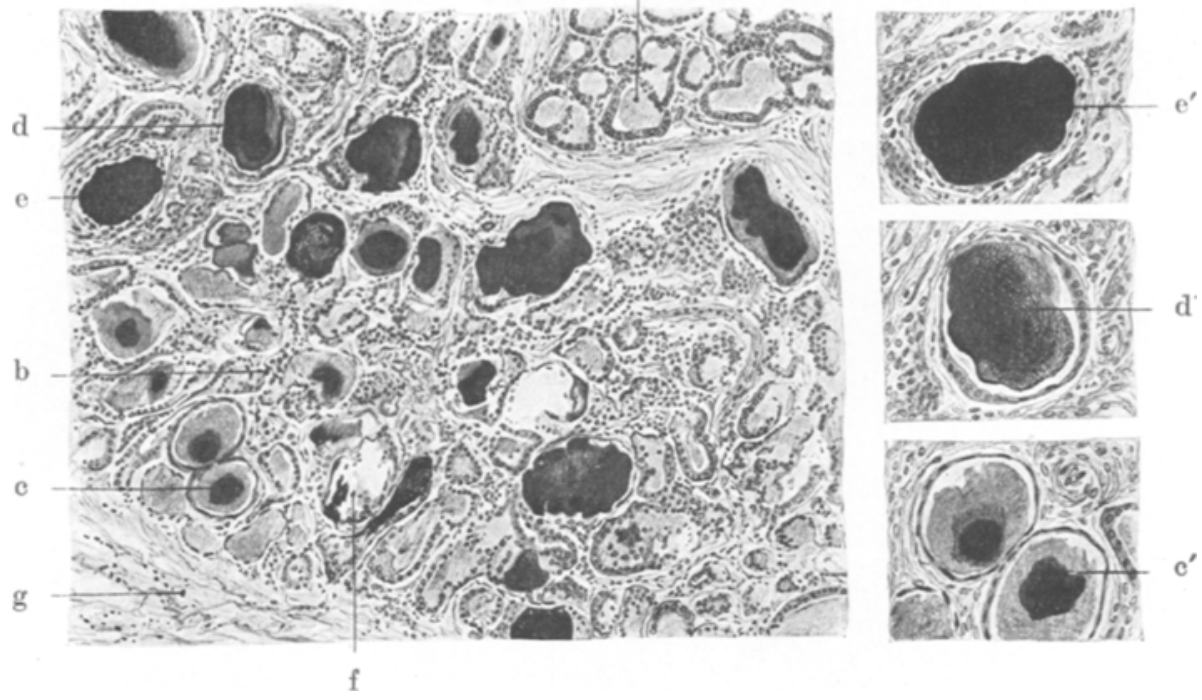

Abb. I. Milchdrüse einer 2 Jahre alten laktierenden Ziege

4 Wochen nach der Thyreoidektomie.

a Normales Milchdrüsenläppchen; b In Veränderung befindliches Milchdrüsenläppchen; c Milchdrüsenalveolen mit beginnender Konkrementbildung (Alveolarepithel noch vollständig vorhanden); $\mathrm{c}^{\prime}$, o bei stärkerer $\nabla$ ergrößerung; $d$ Konkremente in einer Milchdrüsenalveole mit teilweise fehlendem Alveolarepithel; $d$, d bei stärkerer VergröBerung; e Konkremente im intraalveolärem Bindegewebe (Alveolarepithel ganz zugrunde gegangen); e', e bei stärkerer Vergrößerung; f Milchdrüsenalveole mit arusgefallenem Konkrement; g Interlobuläres Bindegewebe.

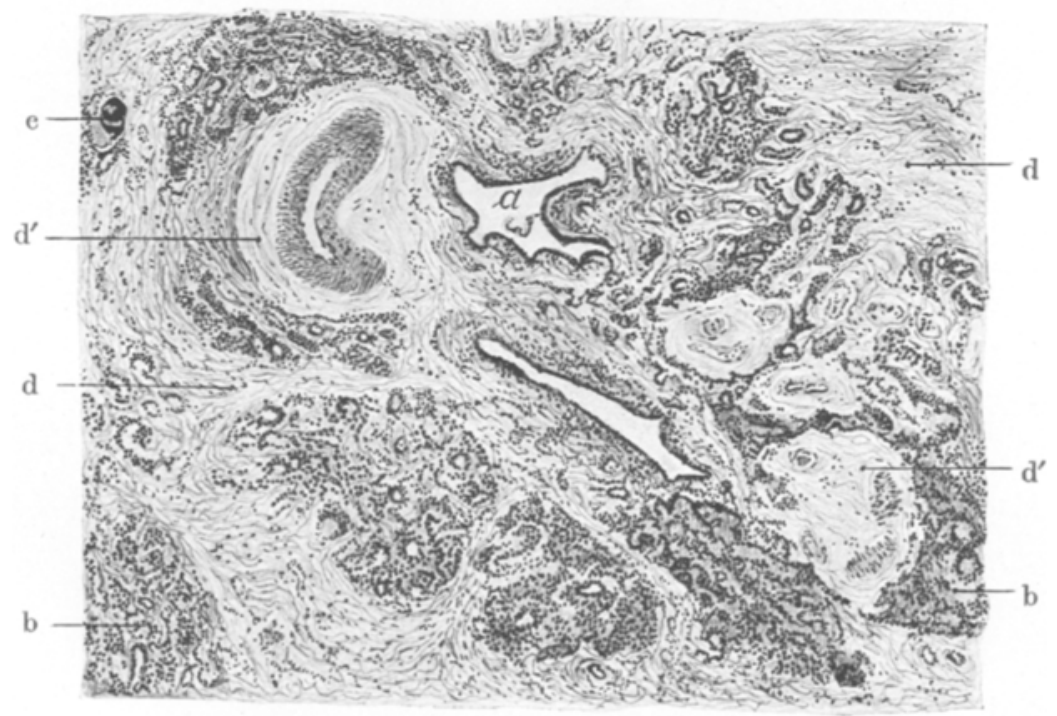

Abb. 2. Milchdrüse einer dreijährigen Ziege zirka 12 Monate nach der Thyreoidektomie. a Zentralkanal (Sekretsammelraum) eines Milchdrüsenläppchens; b Milchdrüsenláppchen; c Konkrement; d. Interlobuläres Bindegewebe; d' Bindegewebige adventitielle Scheiden um Blutgefäße. 


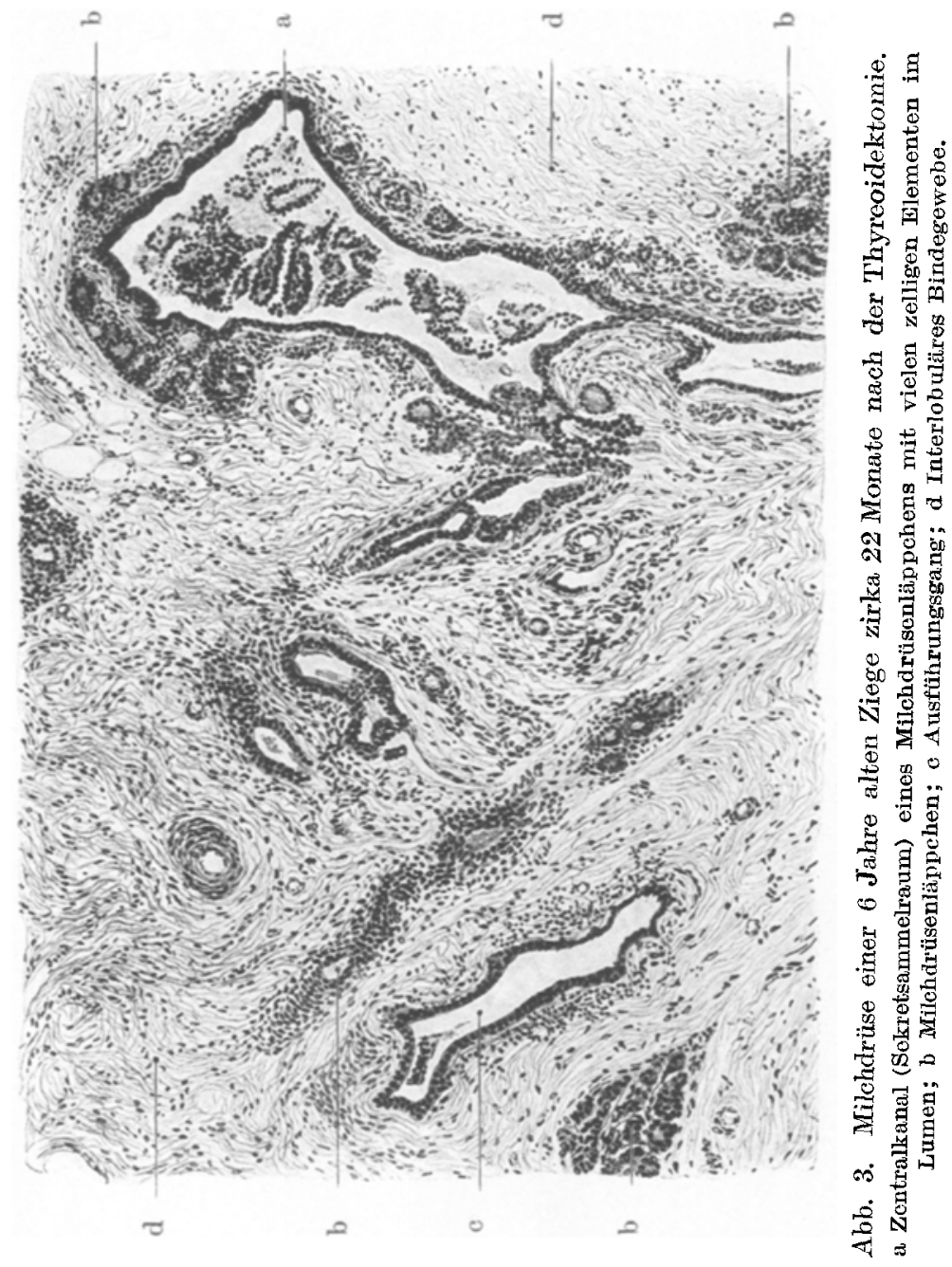

